\title{
Wavelet-based finite element simulation of guided waves containing harmonics
}

\author{
Ambuj Sharma, Sandeep Kumar*, \\ Amit Tyagi and Kumar Kaushik Ranjan
}

\author{
Mechanical Engineering Department, \\ Indian Institute of Technology (BHU), \\ Varanasi, India \\ Email: asharma.rs.mec12@iitbhu.ac.in \\ Email: sandeep.mec@iitbhu.ac.in \\ Email: atyagi.mec@iitbhu.ac.in \\ Email: kkranjan.mec13@itbhu.ac.in \\ *Corresponding author
}

\begin{abstract}
This paper presents a promising numerical scheme for simulation of many harmonics in wave propagation. The wavelet-based adaptive technique eliminates the requirement for a very large number of nodes in finite element method for propagation of such waves. This dynamic adaptive grid selection is based on the fact that very few wavelet coefficients are required to represent a short pulse containing higher harmonics. The method is particularly useful where higher harmonics are ignored due to very high computational cost. In this work, B-spline and Daubechies wavelets-based non-standard (NS) multi-scale operator are applied, and the results are compared with the finite element method.
\end{abstract}

Keywords: non-standard wavelet operator; finite element method; higher harmonics; lamb wave; structural health monitoring.

Reference to this paper should be made as follows: Sharma, A., Kumar, S., Tyagi, A. and Ranjan, K.K. (2019) 'Wavelet-based finite element simulation of guided waves containing harmonics', Int. J. Materials and Structural Integrity, Vol. 13, Nos. 1/2/3, pp.54-66.

Biographical notes: Ambuj Sharma is presently a $\mathrm{PhD}$ student of Mechanical Engineering Department, Indian Institute of Technology (BHU), India. His research interests include wavelet transform, structural health monitoring, and numerical simulation of wave propagation.

Sandeep Kumar is a Professor in the Department of Mechanical Engineering, IIT (BHU), Varanasi. He completed his PhD from the IIT Delhi, India. His research interest is computational mechanics. He is working on wavelet techniques for last ten years.

Amit Tyagi is an Assistant Professor in the Department of Mechanical Engineering, IIT (BHU), Varanasi. He completed his PhD from the IIT (BHU), Varanasi, India. His research interest is mechanical vibration.

Kumar Kaushik Ranjan is presently a $\mathrm{PhD}$ student in the Department of Mechanical Engineering, IIT (BHU), Varanasi. His research interests are wavelet methods and contact analysis. 
This paper is a revised and expanded version of a paper entitled 'Wavelet-based finite element simulation of guided waves containing harmonics' presented at Indian Conference on Applied Mechanics (INCAM) 2017, MNNIT Allahabad, 5-7 July 2017.

\section{Introduction}

Wave propagation can be characterised by a localised region of field variable which changes its space of sharp gradient with time. The conventional finite element method (Langlet et al., 1995; Semblat and Brioist, 2000; Hassan and Veronesi, 2003; Han et al., 2009), which has been preferred for wave propagation, is not suitable to simulate higher harmonics. An adaptive technique is necessary for FEM to avoid a very large number of nodes for numerical simulation of higher harmonics of waves. Recently, customised elements and geometric multi-scale finite element method have been developed to solve various types of wave propagation problems (Kohno et al., 2010; Casadei, 2012; Ham and Bathe, 2012). Multi-scale modelling of FEM must be numerically simple and efficient. A drastic increase of nodes throughout the domain for such problems can be smartly avoided for this highly localised phenomenon using wavelets. The fast, hierarchical and locally adaptive wavelet-based multi-scale algorithms are due to the compactly supported refinable basis functions (Strang and Nguyen, 1996). Wavelets are used for time-frequency analysis (Biswajit, 2007), extensively. Recently, researchers have increased the usage of wavelets for solution of partial differential equations in many different ways (Liandrat and Tchamitchian, 1990; Amaratunga and Williams, 1993; Qian and Weiss, 1993; Carnicer et al., 1996; Sandeep et al., 2011; Quraishi and Sandeep, 2011, 2013). Researchers like Vasilyev and Kevlahan (2005), D'Heedene et al. (2005), and Krysl et al. (2002), etc., could couple the wavelets with finite element and finite difference method after the development of the lifting scheme by Sweldens (1998) and stable completion by Carnicer et al. (1996). The wavelet-based adaptive method has a lot of potential which is not fully utilised by researchers to compete with existing adaptive finite element methods.

The approach in the present work uses an efficient algorithm based on NS wavelet operator initially proposed by Beylkin et al. (1991) for wavelet basis. The algorithm has been generalised and shown for one-dimensional problems by Gines et al. (1998) for wide applications. The paper uses the strength of finite element along with the multi-scale wavelet scheme. To the best knowledge of the authors, so far no researcher has combined the strength of finite element method and efficiency of NS wavelet operator in any numerical simulation. The finest scale finite element field variable of linear or nonlinear problems is projected onto wavelet spaces to get the high- and low-frequency components. A series of projections results in multi-scale decomposition of the fine scale solution into approximate solution (low-frequency components) and successive finer details (high-frequency components). In the proposed multi-scale decomposition, the fine scale solution can be obtained by any other method, but FEM is preferred as it can easily handle complex boundary and loading conditions. Since only some frequency components or wavelets are required to represent higher harmonics, therefore, it provides an effective framework to retain the necessary frequencies of the wave. This wavelet 
transformation is very efficient because it hierarchically filters out significant and insignificant coefficients of the localised burst signal of the large domain. At every time step, the localised time marching forcing function, which is larger than the preset tolerance, is multiplied by the inverse of NS operator which is calculated once at the finest level for the complete domain. The existing orthogonal or biorthogonal wavelet serves the purpose, so the complex process of development of customised wavelet for the operator is not required. Without involving very complex mathematical framework of wavelets, the paper presents a simple and general method which can be applied easily to any other linear or nonlinear problems. The only necessary elements of the wavelets in combination with algorithm are provided to facilitate adaptive grid techniques.

\section{Mathematical formulation}

\subsection{Elastic wave}

In this study, we considered the governing equation of elastic waves of plane strain that exist in free plates:

$$
\begin{aligned}
& C_{L}^{2} \frac{\partial^{2} u}{\partial x^{2}}+\left(C_{L}^{2}-C_{T}^{2}\right) \frac{\partial^{2} v}{\partial x \partial y}+C_{T}^{2} \frac{\partial^{2} u}{\partial y^{2}}+f_{x}=\frac{\partial^{2} u}{\partial t^{2}}, \\
& C_{L}^{2} \frac{\partial^{2} v}{\partial y^{2}}+\left(C_{L}^{2}-C_{T}^{2}\right) \frac{\partial^{2} u}{\partial x \partial y}+C_{T}^{2} \frac{\partial^{2} v}{\partial x^{2}}+f_{y}=\frac{\partial^{2} v}{\partial t^{2}},
\end{aligned}
$$

$C_{L}^{2}=\lambda+2 \mu / \rho$ and $C_{T}^{2}=\mu / \rho$ are longitudinal velocity and shear velocity, respectively. $\lambda=E v /(1+v)(1-2 v)$ and $\mu=E / 2(1+v)$ are Lame's constants. $E$ and $v$ are Young's modulus and Poisson ratio, respectively. Figure 1 shows displacement $u$ in the direction of wave propagation (x-direction) and $v$ through the thickness (y-direction).

The 2D plain strain problem is discretised into the set of finite element equations as:

$$
[K]+[u]+[M][\ddot{u}]=0
$$

where $[K]$ is the global stiffness matrix and $[M]$ is the global mass matrix, $[u]$ and $[\ddot{u}]$ are unknown coefficient vectors. Implicit Newmark time integration scheme is used to solve dynamic equation (2a). The marching variable $[u]_{s+1}$ of the dynamic equation at time $t_{s+1}$ can be obtained from the coefficients $[u]_{s}$ at $t_{s}$ by using the recursive relation of $\alpha$-family of approximation (Reddy, 2005) and expressed as:

$$
[\hat{K}]_{[u]_{s+1}}=[\tilde{K}]_{[u]_{s}}
$$

Figure 1 Plate geometry and coordinates

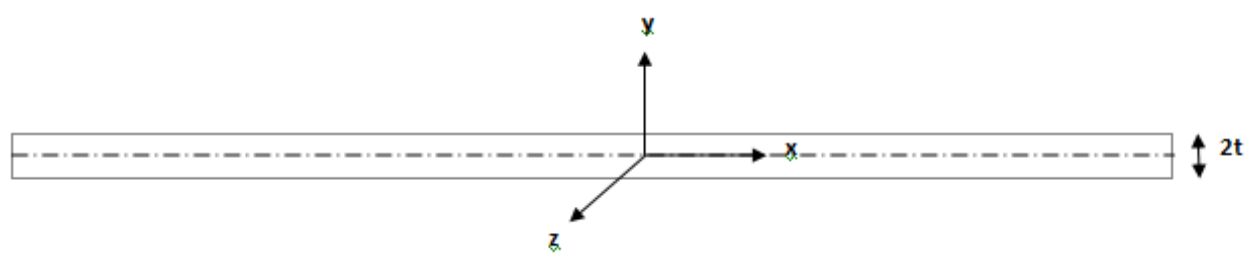




\subsection{Multi-scaling using wavelets}

The concept of multi-scale analysis is to interpolate an unknown field at a coarse level by means of so-called scaling functions. Any improvement to the initial approximation is achieved by adding 'details' provided by new functions known as wavelets. A multi-scaling analysis forms a sequence of closed subspaces so that (Mallat, 1998)

$$
V_{0} \subset V_{1} \subset V_{2} \subset \ldots L^{2}(R)
$$

satisfying the following properties:

$$
\begin{aligned}
& f(x) \in V_{j} \Leftrightarrow f(2 x) \in V_{j+1} \\
& f(x) \in V_{0} \Leftrightarrow f(x+1) \in V_{0} \\
& \overline{\cup V_{j}} \text { is dense in } L^{2}(R) ; \cap V_{j}=\{0\} .
\end{aligned}
$$

Each subspace $V_{j}$ is spanned by a set of scaling function $\left\{\varphi_{j, k}(x), \forall k \in Z\right\}$. The complement of $V_{j}$ in $V_{j+1}$ is defined as subspace $W_{j}$ such that

$$
V_{j+1}=V_{j} \oplus W_{j} \quad \forall j \in Z .
$$

The space $V_{j+1}$ can be decomposed in a consecutive manner as:

$$
V_{j+1}=V_{0} \oplus W_{0} \oplus W_{1} \oplus W_{2} \oplus \ldots \oplus W_{j}
$$

The basis functions in $W_{j}$ are called wavelet functions and are denoted by $\varphi_{j, k}$. These wavelet and scaling functions in different scales are used for wavelet-based multi-scaling. Approximating a function $f \in L^{2}(R)$ by its projection $P_{j} f$ onto the space $V_{j}$ and the projection of $f$ on $W_{j}$ as $Q_{j} f$, we have

$$
P_{j} f=P_{j-1} f+Q_{j-1} f \text {. }
$$

If the coefficient vector of $P_{j} f$ is $u_{j}=\left\{u_{j, 0}, \ldots, u_{j, v(j)}\right\}^{T}$ and coefficient vector of $Q_{j} f$ is $d_{j}=\left\{d_{j, 0}, \ldots, d_{j, w(j)}\right\}^{T}$ then two levels of resolution $(j$ and $j-1)$ in spatial domain can be expressed using wavelet transform as:

$$
u_{j}=\left[Q_{j-1} \mid P_{j-1}\right]\left[\frac{d_{j-1}}{u_{j-1}}\right],
$$

where $P_{j}$ is a $\eta(j) \times \eta(j-1)$ matrix and $Q_{j}$ is a $\omega(j) \times \omega(j-1)$ matrix. $\eta(j)$ and $\omega(j)$ are dimensions of $V_{j}$ and $W_{j}$ spaces, respectively. After transformation of fine scale $u_{j}$, we get coarse scale $u_{j-1}$ which is a kind of average and detail coefficients $d_{j-1}$ which is a kind of difference between fine scale and average coefficients. The insignificant detail coefficients in the smooth region are eliminated to reduce the grid points. In this paper, B-spline wavelet and Daubechies (D4) wavelet (Stollnitz et al., 1996) are used for wave propagation.

The synthesis matrices $P_{j}$ and $Q_{j}$ for D4 are: 


$$
\mathbf{P}_{j}=\left[\begin{array}{c}
\alpha_{1}, \alpha_{2}, \alpha_{3}, \alpha_{4}, 0,0, \ldots, 0 \\
0,0, \alpha_{1}, \alpha_{2}, \alpha_{3}, \alpha_{4}, 0,0, \ldots, 0 \\
0,0,0,0, \alpha_{1}, \alpha_{2}, \alpha_{3}, \alpha_{4}, 0,0, \ldots, 0 \\
\vdots \\
\vdots \\
0,0, \ldots, 0, \alpha_{1}, \alpha_{2}, \alpha_{3}, \alpha_{4} \\
\alpha_{3}, \alpha_{4}, 0,0, \ldots, 0, \alpha_{1}, \alpha_{2}
\end{array}\right]
$$

$$
\mathbf{Q}_{j}=\left[\begin{array}{c}
\beta_{1}, \beta_{2}, \beta_{3}, \beta_{4}, 0,0, \ldots, 0 \\
0,0, \beta_{1}, \beta_{2}, \beta_{3}, \beta_{4}, 0,0, \ldots, 0 \\
0,0,0,0, \beta_{1}, \beta_{2}, \beta_{3}, \beta_{4}, 0,0, \ldots, 0 \\
\vdots \\
\vdots \\
0,0, \ldots, 0, \beta_{1}, \beta_{2}, \beta_{3}, \beta_{4} \\
\beta_{3}, \beta_{4}, 0,0, \ldots, 0, \beta_{1}, \beta_{2}
\end{array}\right]
$$

The constants $\alpha_{i}$ and $\beta_{i}$ are defined as:

$$
\begin{array}{ll}
\alpha_{1}=\frac{1+\sqrt{3}}{4 \sqrt{2}}, & \alpha_{2}=\frac{3+\sqrt{3}}{4 \sqrt{2}} \\
\alpha_{3}=\frac{3-\sqrt{3}}{4 \sqrt{2}} & \alpha_{4}=\frac{1-\sqrt{3}}{4 \sqrt{2}} \\
\beta_{1}=\frac{1-\sqrt{3}}{4 \sqrt{2}} & \beta_{2}=\frac{\sqrt{3}-3}{4 \sqrt{2}} \\
\beta_{3}=\frac{3+\sqrt{3}}{4 \sqrt{2}} & \beta_{4}=\frac{-1-\sqrt{3}}{4 \sqrt{2}}
\end{array}
$$

\subsection{Non-standard operator}

Multi-scale is performed by projecting the solution at the fine scale space $V_{j}$ onto next coarser levels $W_{j-1}$ and $V_{j-1}$, recursively. The finite element method with Lagrange interpolation function as a basis function is used to calculate the stiffness matrix. Let the finite element equation $(2 \mathrm{~b})$ at the finest resolution is expressed as:

$$
A_{j} u_{j}=f_{j}
$$

Here subscript $j$ of the equation represents the level of resolution. Using wavelets for forward transformation $T_{j}=\left[Q_{j} \mid P_{j}\right]$ the finest scale is replaced by multi-scale as shown in the Figure 2, which can be expressed as:

$$
T_{j-1}\left[\frac{d_{j-1}}{u_{j-1}}\right]=u_{j},
$$

substituting equation (10) in equation (9), we get 


$$
\begin{aligned}
& A_{j} T_{j-1}\left[\frac{d_{j-1}}{u_{j-1}}\right]=f_{j}, \\
& \text { or } T_{j-1}^{T} A_{j} T_{j-1}\left[\frac{d_{j-1}}{u_{j-1}}\right]=T_{j-1}^{T} f_{j}
\end{aligned}
$$

The multi-scale solution of finer scale can be expressed as:

$$
A_{j-1}\left[\frac{d_{j-1}}{u_{j-1}}\right]=f_{j-1}
$$

where $u_{j-i}$ are the scaling coefficients, $d_{j-i}$ are the wavelets coefficient at the lower resolution. For 2D problem, the equation (9) can be expanded as:

$$
\left[\begin{array}{ll}
{\left[k_{j}^{u u}\right]} & {\left[k_{j}^{u v}\right]} \\
{\left[k_{j}^{v u}\right]} & {\left[k_{j}^{v v}\right]}
\end{array}\right]\left[\begin{array}{l}
u_{j} \\
v_{j}
\end{array}\right]=\left[\begin{array}{l}
{\left[f_{j}^{u}\right]} \\
{\left[f_{j}^{v}\right]}
\end{array}\right]
$$

After application of wavelet transform, the standard operator is obtained as:

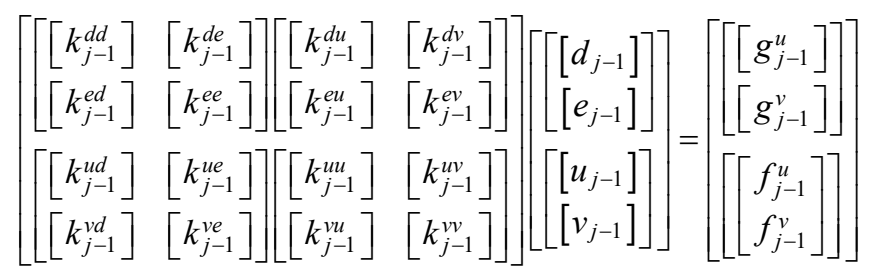

where $d_{j-1}$ and $e_{j-1}$ are detail coefficients corresponding to the scaling coefficients $u_{j-1}$ and $v_{j-1}$. The solution can be transformed back in terms of nodal displacement by inverse wavelet transform. Similarly, refinement at the next level can be expressed as:

$$
T_{j-2}^{T} A_{j-1} T_{j-2}\left[\frac{\frac{d_{j-2}}{d_{j-1}}}{u_{j-2}}\right]=T_{j-2}^{T}\left[\frac{g_{j-1}}{f_{j-1}}\right]
$$

The wavelet transform can be continued until a desired low and high-frequency component representation is achieved. The well known standard operator [equation (15)] can be easily represented in the form of NS operator as shown in Figure 3 (Beylkin et al., 1991). It has been proved by Gines et al. (1998) that NS operator is more efficient than the standard form of the operator. The product of NS operator with its inverse is the non-standard identity matrix which is shown in Figure 3 for a three-level transform. The LU decomposition of the operator for computation of inverse is shown in Figure 4.

The solution at any time step is obtained by multiplication of NS form of inverse with the NS form of the right side vector of Newmark scheme [equation 2(b)]. In this process, the inverse of the NS operator for big finite element coefficient matrix at the finest level for the complete domain is calculated only once, i.e., there is no need of re-meshing and recomputing the operator with a change of sharp gradient in space and time. To get higher harmonics with the least number of detail coefficients, the forces which are less than a preset limit at the fine scale are assumed as zeros. Though the whole domain is 
discretised with very fine mesh to simulate higher harmonics yet the computation of matrix-vector product at every time step is very economical. It is economical at coarse scale due to a small size of the matrix. At the fine scale, the very small size of non-zero forcing function cuts down the computational cost.

Figure 2 Multi-scale decomposition, (a) space $V_{j}$ (b) space $V_{j-1} \oplus W_{j-1}$ (c) space $V_{j-2} \oplus W_{j-2}$ $\oplus W_{j-1}$ (see online version for colours)

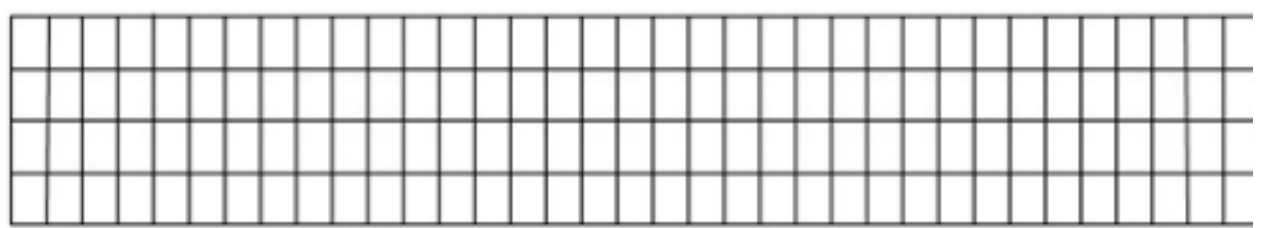

(a)

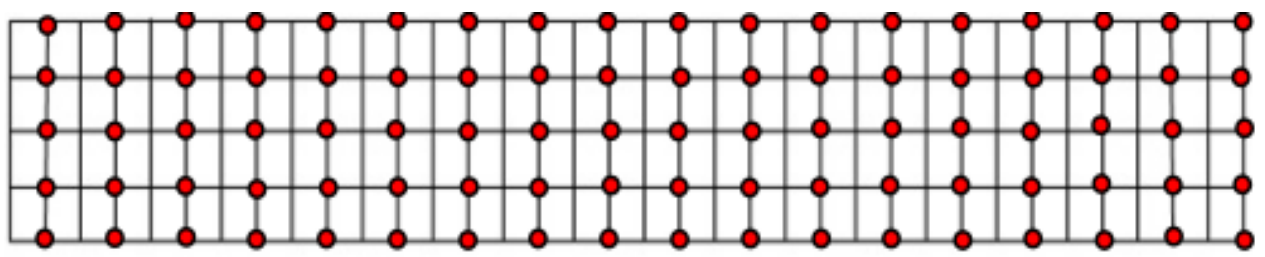

(b)

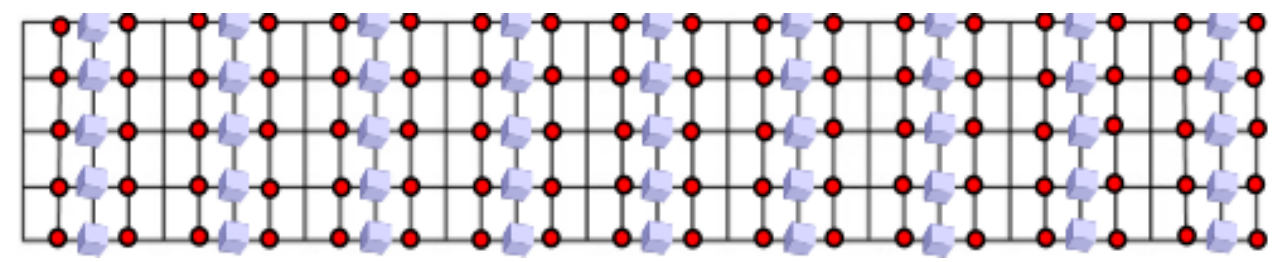

(c)

Figure 3 The product of NS operator with its inverse

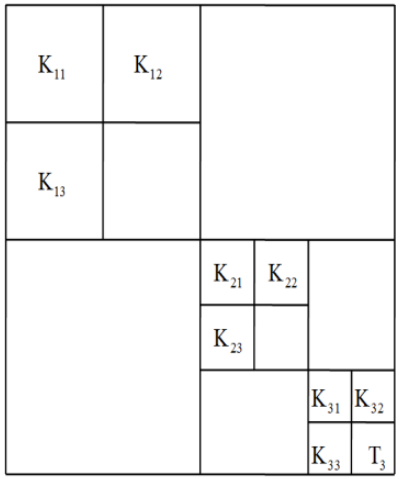

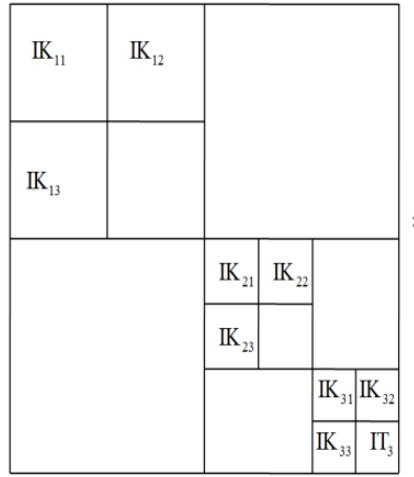

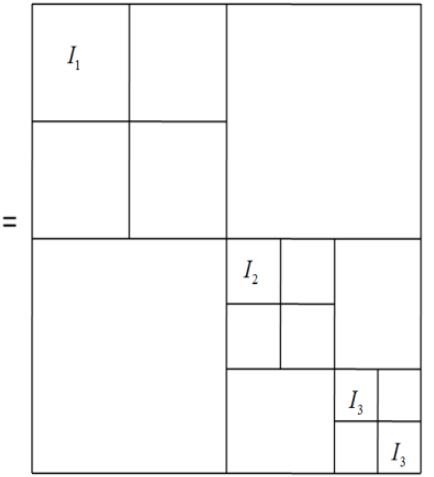


Figure 4 LU decomposition of NS operator (see online version for colours)
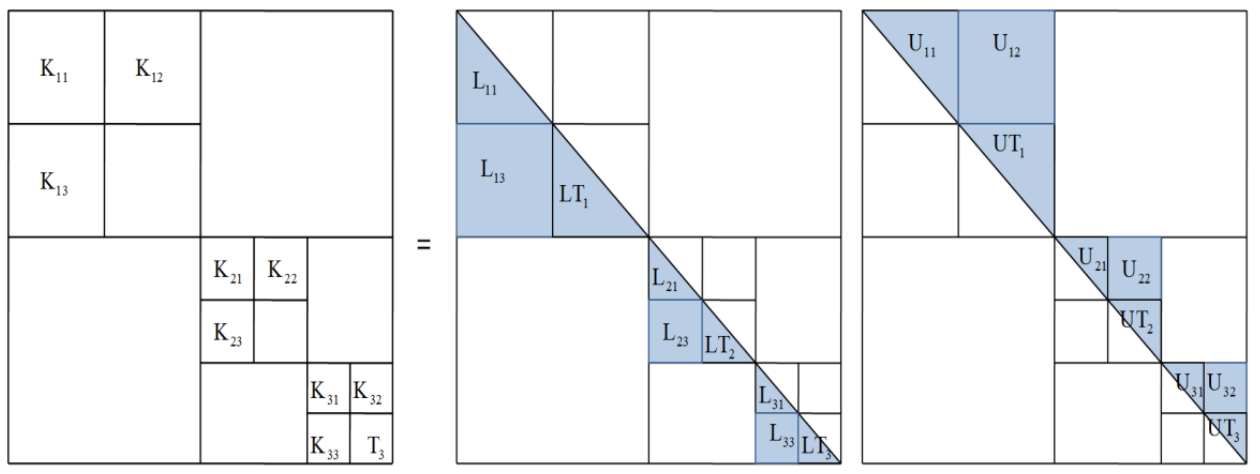

Figure 5 Computational mesh used in two dimensional (see online version for colours)

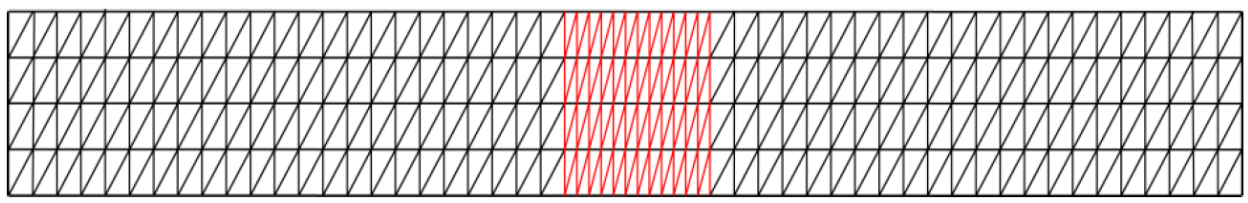

The change in the position of the sharp gradient will only change the forcing function. To reduce error due to end effects, the computational grid should include points near the sharp gradient zone also. In this process, we include those grid points whose wavelets coefficients will become significant during the next moving step of the wave. Figure 5 represents the adaptive mesh where detail coefficients at high gradient zone are retained. As shown in Figure 5, constant strain triangles with uniformly distributed nodes throughout the plate and four elements along the thickness are used.

\section{Results and discussion}

The computational cost of simulation of waves with higher harmonics is very high. The objective of the method is to propagate higher harmonics of waves using least number of nodes. In this experiment, we considered Lamb wave which is frequently used for detection of a crack in plates. Some higher harmonics are added in the Lamb wave to see the effectiveness of the proposed method in the simulation of wave propagation. Figure 6(a) depicts the five cycles modulated sinusoidal Hanning window excitation signal of linear Lamb wave at $400 \mathrm{kHz}$. These Lamb waves have been used for detection of cracks in the plate like structures. This study uses the following actuation function with $400 \mathrm{kHz}$ central frequency driven through a Hanning window:

$$
F(t)=\left\{\begin{array}{cc}
A_{0} \sin (\omega t)\left(\sin \left(\frac{\omega t}{10}\right)\right)^{2}+\frac{A_{0}}{10} \sin (10 \omega t) *(\sin (\omega t))^{2}, & t<\frac{10 \pi}{\omega} \\
0, & \text { otherwise }
\end{array}\right.
$$


Figure 6 Excitation signal (a) Lamb wave and (b) Lamb wave with higher harmonics (see online version for colours)

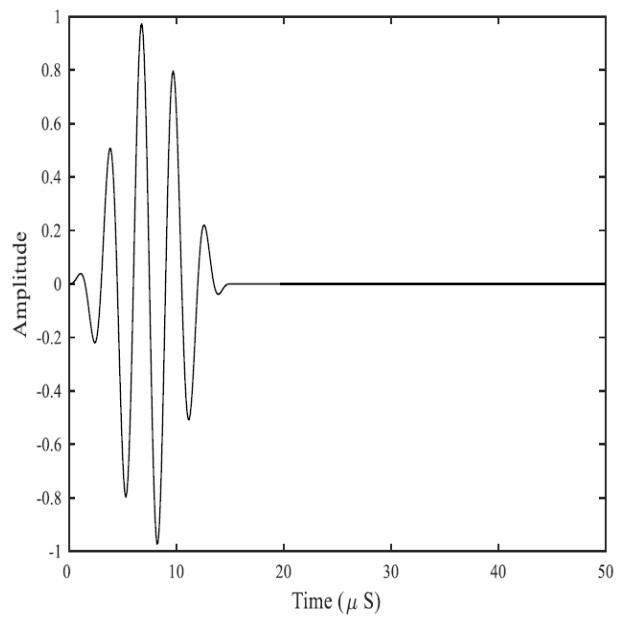

(a)

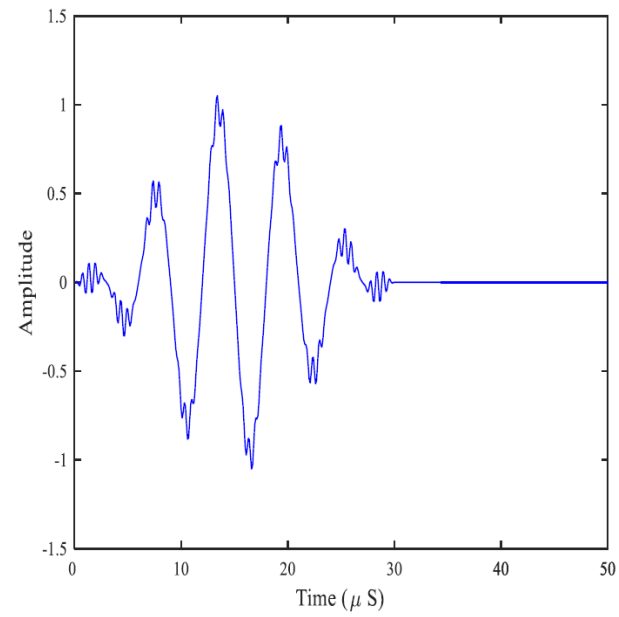

(b)

Figure 7 Wave propagation for $400 \mathrm{kHz}$ central frequency in plate structure at different nodes using 20 elements per wavelength (see online version for colours)

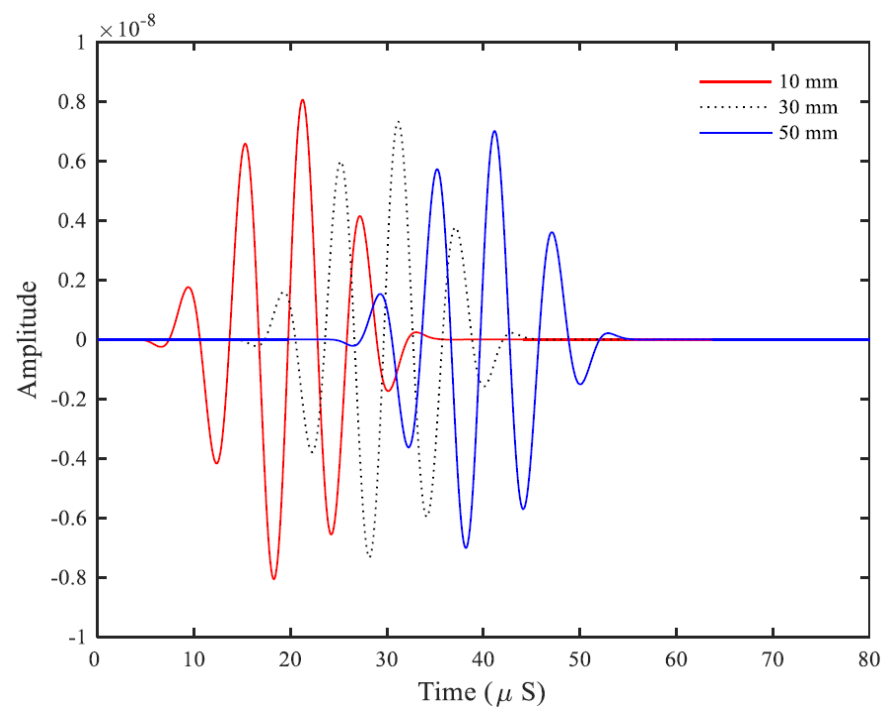

The excitation signal on a plate with a higher harmonics is shown in Figure 6(b). An aluminium plate of $500 \mathrm{~mm}$ length and $1 \mathrm{~mm}$ thickness is considered in the analysis. The material properties are assumed as Poisson's ratio $=0.3$, density $=2,700 \mathrm{~kg} / \mathrm{m}^{3}$, and Young's modulus $\mathrm{E}=69 \mathrm{GPa}$. The Lamb wave in this material has longitudinal velocity $C_{L}=5,299.1 \mathrm{~m} / \mathrm{s}$ and transverse velocity $C_{T}=3,135 \mathrm{~m} / \mathrm{s}$. The waves are actuated by employing pin forces applied to the left boundary of the plate. The excitation forces are perpendicular to the longitudinal (propagating) direction. In-phase pin forces are applied to the top and bottom edge nodes of the plate for excitation of fundamental symmetric 
$\left(\mathrm{S}_{0}\right)$ modes, and the anti-symmetric modes are propagated by imposing out-of-phase pin forces (Nieuwenhuis et al., 2005). In this paper, we considered a case in which the pure $\mathrm{S}_{0}$ mode is excited. Five cycles Hanning window excitation signal is applied to provide a limited cycle sinusoidal tone burst.

Figure 8 Response of plate structure for (a) 50 elements per wavelength and (b) 100 elements per wavelength (see online version for colours)

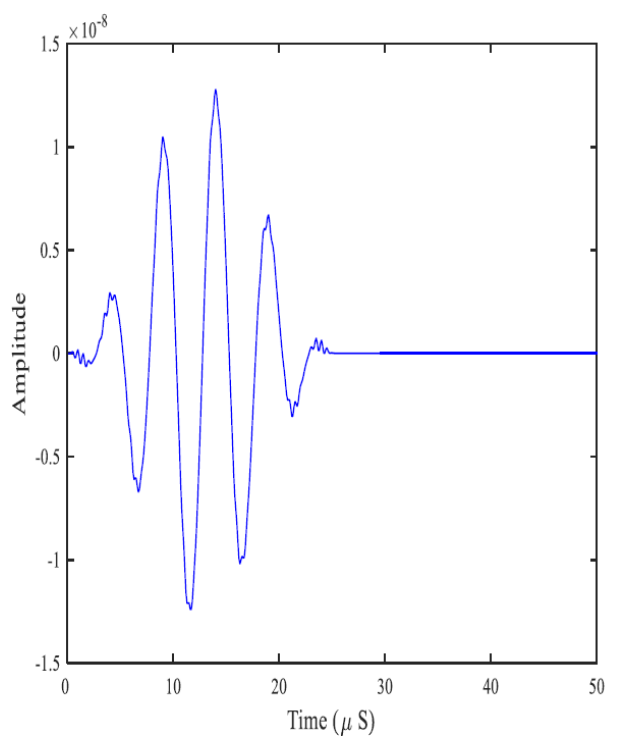

(a)

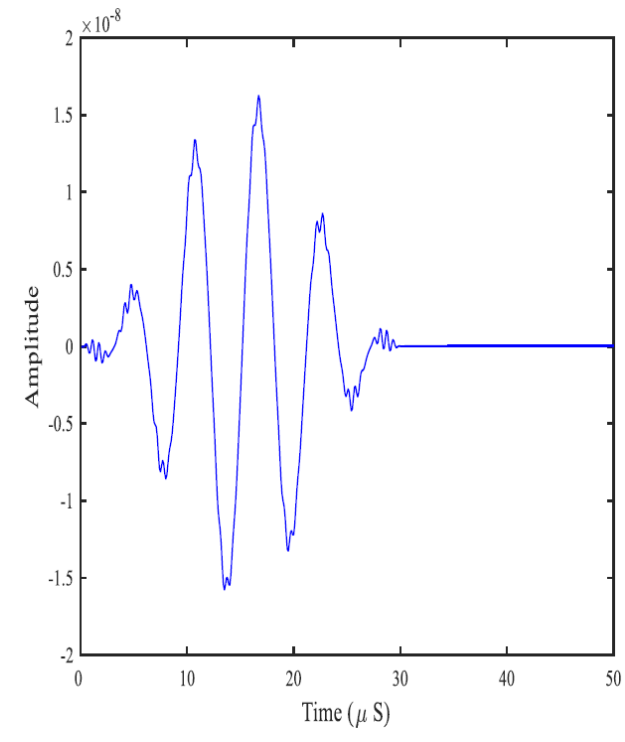

(b)

Lamb wave propagation signals on a plate at different nodes at the distance of 10, 30 and $50 \mathrm{~mm}$ from the left end, generated by FEM, are illustrated in Figure 7. It can be observed that wave is propagated, but there is some attenuation. In general, 20 elements per wavelength are required in the case of Lamb wave, but this is not sufficient for higher harmonic simulation. Higher frequency wave propagation problems demand enormous computer resources because of highly dense mesh and a considerable number of time integration steps. Figure 8(a) depicts the nodal displacement obtained using FE simulation of the plate with 50 elements per wavelength. It can be observed that higher harmonics are not properly visible. On the other hand, as shown in Figure 8(b), higher harmonics are visible for 100 elements per wavelength.

In the present analysis, B-spline and Daubechies (D4) wavelet are used to establish robustness and sensitivity of wavelet-based wave propagation method. To capture higher harmonics in the plates, FEM uses 17,080 uniformly distributed nodes while half of the FEM nodes are required after application of one level of wavelet transform. The response of plate received from B-spline and D4 wavelet transform at level-1 along with FEM results are demonstrated in Figure 9(a). It establishes a good agreement between the conventional finite element and the proposed method. It can be observed that B-spline wavelet produces response close to FEM results, while there is some deviation in the results of D4 wavelet. Further, we examined wavelet-based method at the various levels of wavelet transform to find the level up to which this method can work efficiently. In 
next wavelet transform level-2, the number of uniformly distributed nodes is reduced to 4,270 which are one-fourth of the FEM nodes, and the results are shown in Figure 9(b). Similarly, wavelet transform level-3 is utilising only 2,135 , i.e., one-eighth of the FEM nodes. The resulting wavelet response and FEM response of the plate are shown in Figure 9(c). These results show some attenuation, but wavelets are not eliminating higher frequency components of waves which are important in many analysis. The wavelet transform level-4 uses only 1,068 nodes which are one-sixteenth of the FEM nodes. As shown in Figure 9(d), such a small number of nodes are also sufficient to observe higher harmonics. It is evident as low as $6.25 \%$ of FEM nodes are enough to propagate higher harmonics when the proposed wavelet-based method is used.

Figure 9 Comparison of plate response at $400 \mathrm{kHz}$ central frequency, (a) wavelet transform level-1 (b) wavelet transform level-2 (c) wavelet transform level-3 (d) wavelet transform level-4 (see online version for colours)

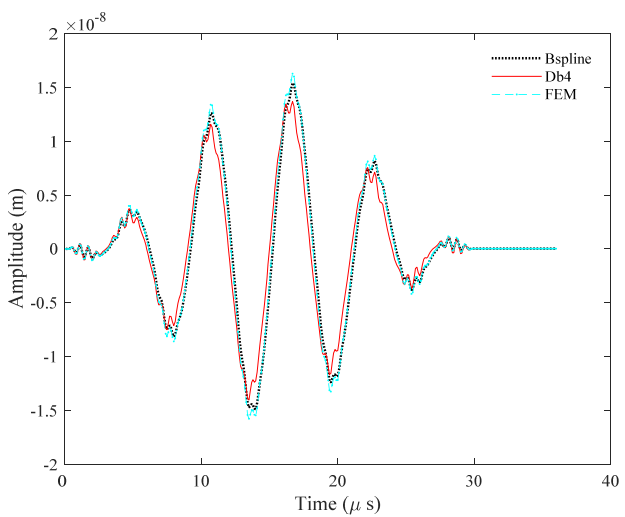

(a)

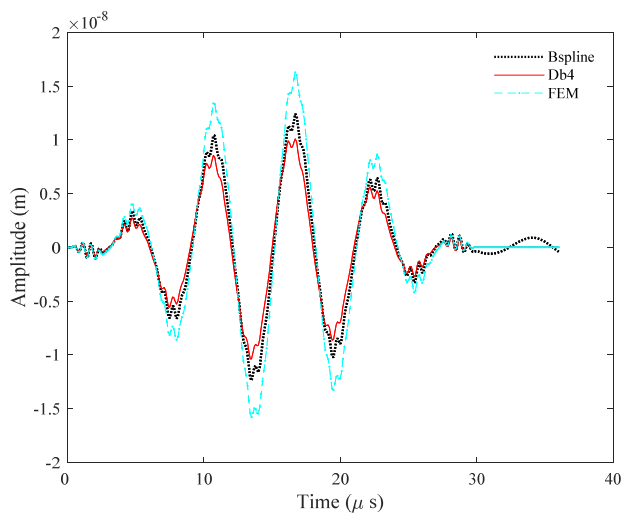

(c)

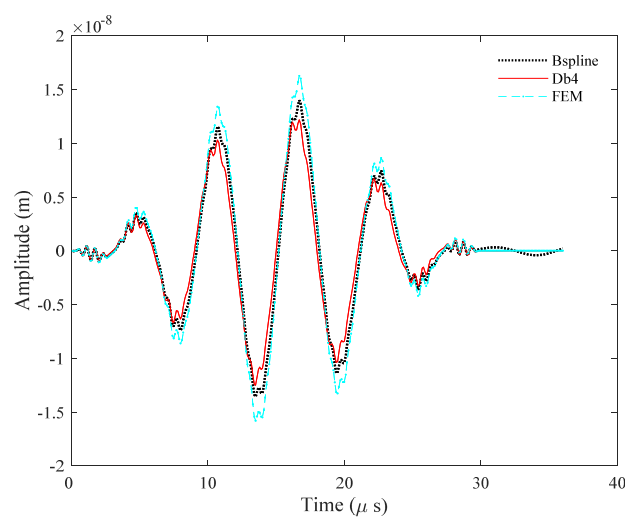

(b)

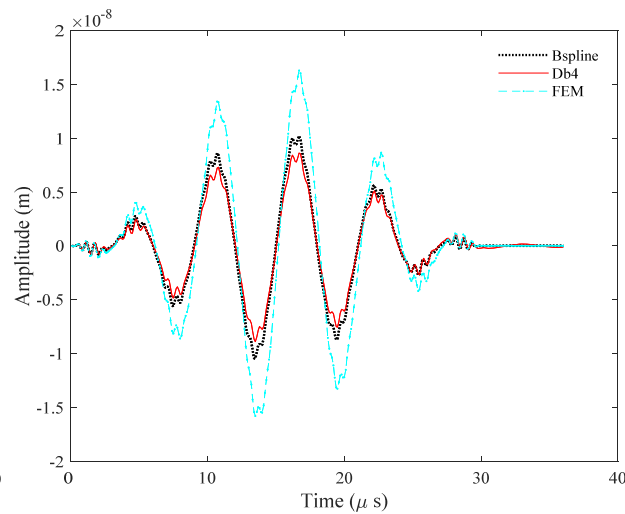

(d)

\section{Conclusions}

The localisation properties and multi-level structure of the wavelets in the physical space with the NS operator are proved to be an efficient tool in developing the adaptive 
computational approach. We presented a wavelet-based framework to reduce the size of finite element stiffness matrix which is becoming too large in the case of nonlinear wave propagation problem. Although the tone burst signals have very localised nature, they require very fine grid in the whole domain to simulate higher harmonics. Without disturbing higher harmonics, the proposed method is able to reduce the computational cost significantly. In this paper, B-splines and Daubechies wavelets are used, but other wavelets can also be used. Some attenuation of the waves are not affecting the higher harmonics, so it will not disturb the analysis. This approach has many simple and advantageous features drawing from both conventional finite element and wavelet methods. Without disturbing the programming advantages of FEM regarding the implementation of boundary conditions and efficient numerical integration of interpolation functions, wavelet-based methods are able to reduce the size of the matrix as much as one by sixteenth of original FE matrix. These fundamental characteristics show that the wavelet-based method can be utilised for more complex wave propagation problems.

\section{References}

Amaratunga, K. and Williams, J.R. (1993) 'Wavelet based Green's function approach to 2D PDEs', Engineering Computations, Vol. 10, No. 4, pp.349-367.

Beylkin, G., Coifman, R. and Rokhlin, V. (1991) 'Fast wavelet transforms and numerical algorithms I', Communications on Pure and Applied Mathematics, Vol. 44, No. 2, pp.141-183.

Biswajit, B. (2007) 'Assessment of structural integrity via wavelet based time-frequency analysis of vibration signals', International Journal of Materials and Structural Integrity, Vol. 1, Nos. 1/2/3, pp.238-258.

Carnicer, J.M., Dahmen, W. and Peña, J.M. (1996) 'Local decomposition of refinable spaces and wavelets', Applied and Computational Harmonic Analysis, Vol. 3, No. 2, pp.127-153.

Casadei, F. (2012) Multiscale Analysis of Wave Propagation in Heterogeneous Structures, dissertation, School of Aerospace Engineering, Georgia Institute of Technology.

D’Heedene, S., Amaratunga, K. and Castrillón-Candás, J. (2005) 'Generalized hierarchical bases: a wavelet-Ritz-Galerkin framework for Lagrangian FEM', Engineering Computations, Vol. 22, No. 1, pp.15-37.

Gines, D., Beylkin, G. and Dunn, J. (1998) 'LU factorization of non-standard forms and direct multiresolution solvers', Applied and Computational Harmonic Analysis, Vol. 5, No. 2, pp.156-201.

Ham, S. and Bathe, K.J. (2012) 'A finite element method enriched for wave propagation problems', Computers \& Structures, Vols. 94-95, pp.1-12.

Han, S., Palazotto, A.N. and Leakeas, C.L. (2009) 'Finite element analysis of lamb wave propagation in a thin aluminum plate', Journal of Aerospace Engineering, Vol. 22, No. 2, pp.185-197.

Hassan, W. and Veronesi, W. (2003) 'Finite element analysis of Rayleigh wave interaction with finite-size, surface-breaking cracks', Ultrasonics, Vol. 41, No. 1, pp.41-52.

Kohno, H., Bathe, K.J. and Wright, J.C. (2010) 'A finite element procedure for multiscale wave equations with application to plasma waves', Computers \& Structures, Vol. 88, Nos. 1-2, pp.87-94.

Krysl, P., Grinspun, E. and Schroder, P. (2002) 'Natural hierarchical refinement for finite element methods', International Journal for Numerical Methods in Engineering, Vol. 56, No. 8, pp.1109-1124. 
Langlet, P., Hladky-Hennion A.C. and Decarpigny, J.N. (1995) 'Analysis of the propagation of plane acoustic waves in passive periodic materials using the finite element method', The Journal of the Acoustical Society of America, Vol. 98, No. 5, pp.2792-2800.

Liandrat, J. and Tchamitchian, P.H. (1990) Resolution of the 1D Regularized Burgers Equation using a Spatial Wavelet Approximation, NASA Langley Research Centre, Hampton VA, Report No: NASA CR-187480.

Mallat, S. (1998) A Wavelet Tour of Signal Processing, 3rd ed., Academic Press, San Diego.

Nieuwenhuis, J.H., Neumann, J.J., Greve, D.M. and Oppenheim I.J. (2005) 'Generation and detection of guided waves using PZT wafer transducers', IEEE Trans. Ultrason., Ferroelect., Freq. Control., Vol. 52, No. 11, pp.2103-2111.

Qian, S. and Weiss, J.J. (1993) 'Wavelets and the numerical solution of partial differential equations', Journal of Computational Physics, Vol. 106, No. 1, pp.155-175.

Quraishi, S.M. and Sandeep, K. (2011) 'A second generation wavelet based finite elements on triangulations', Computational Mechanics, Vol. 48, No. 2, pp.63-174.

Quraishi, S.M. and Sandeep, K. (2013) 'Multiscale modeling of beam and plates using customized second-generation wavelets', Journal of Engineering Mathematics, Vol. 83, No 1, pp.185-202.

Reddy, J.N. (2005) An Introduction to the Finite Element Method, 3rd ed., Tata McGraw-Hill Education, India.

Sandeep, K., Gaur, S., Dutta, D. and Kushwaha, H.S. (2011) 'Wavelet based schemes for linear advection-dispersion equation', Applied Mathematics and Computation, Vol. 218, No. 7, pp.3786-3798.

Semblat, J. and Brioist, J. (2000) 'Efficiency of higher order finite elements for the analysis of sesmic wave propagation', Journal of Sound Vibration, Vol. 231, pp.460-467.

Stollnitz, E.J., Derose, T.D. and Salesin, D.H. (1996) Wavelets for Computer Graphics, Morgan Kaufmann Pub. Inc., California.

Strang, G. and Nguyen, T. (1996) Wavelets and Filter Banks, Wellesley-Cambridge Press, Wellesley, MA.

Sweldens, W. (1998) 'The lifting scheme: a construction of second generation wavelets', SIAM Journal of Mathematical Analysis, Vol. 29, No. 2, pp.511-546.

Vasilyev, O.V. and Kevlahan, N.K-R. (2005) 'An adaptive multilevel wavelet collocation method for elliptic problems', Journal of Computational Physics, Vol. 206, No. 2, pp.412-431. 